\title{
Towards Life Long Learning: Three Models for Ubiquitous Applications
}

\author{
Andreas Holzinger ${ }^{1 *}$, Alexander Nischelwitzer ${ }^{2}$, Silvia Friedl ${ }^{2}$ and $\mathrm{Bo} \mathrm{Hu}^{3}$ \\ ${ }^{1}$ Institute for Medical Informatics, Statistics and Documentation, Medical University Graz, Austria \\ ${ }^{2}$ School of Information Management, University of Applied Sciences Graz, Austria \\ ${ }^{3}$ School of Electronics and Computer Science, University of Southampton, United Kingdom
}

\section{Summary}

In this paper, we present three experimental proof-of-concepts: First, we demonstrate a Ubiquitous Computing Framework (UCF), which is a network of interacting technologies that support humans ubiquitously. We then present practical work based on this UCF framework: TalkingPoints, which was originally developed for use at trading fairs in order to identify each participant and company via transponder and provide specific information during and after use. Finally, we propose GARFID, a concept for using advanced technologies for teaching young children. The main outcome of this research is that the concept of UCF raises a lot of possibilities, which can bring value and benefits for end-users. When one follows the Working-is-Learning paradigm, it can be seen that the implementation of this type of technology can support Life Long Learning, thereby providing evidence that technology can benefit everybody and make life easier.

Copyright (c) 2008 John Wiley \& Sons, Ltd.

KEY WORDS: Ubiquitous Computing Framework; Life Long Learning; RFID Application

\section{Introduction and Motivation for Research}

Ubiquitous and pervasive computing technology is a challenging and booming research area and has a number of significant advantages over traditional technologies [52]. Today, tiny embedded devices attached to everyday objects can equip them with sensing, perception, computation, and communication abilities [10]; most interesting, the deployment of such ubiquitous technology on a larger scale is about to become both technically and economically feasible [4]. In other words, it is becoming more

${ }^{*}$ Correspondence to: Research Unit HCI4MED, Institute for Medical Informatics, Statistics and Documentation, Medical University Graz, A-8036 Graz, Auenbruggerplatz 2/V, Austria, EMail: andreas.holzinger@meduni-graz.at $\dagger$

Copyright (C) 2008 John Wiley \& Sons, Ltd. Prepared using wcmauth.cls [Version: 2007/01/09 v1.00] affordable and consequently its availability in daily use is increasing [3]. It could be said, that the vision of M. Weiser [48] has definitely come true: "The most profound technologies are those that disappear. They weave themselves into the fabric of everyday life until they are indistinguishable from it". Small microcomputers can be embedded in everyday objects all around us, using wireless connections. Such a network of mobile and fixed devices can do things for us unobtrusively; a end-user would only notice the device's (hopefully positive) effects. Weiser called such systems calm technology, due to the fact that such technologies make it easier for us to focus and concentrate on our actual activities, rather than forcing us to interact with and operate the devices, which is often an awkward process - typical of 
standard Personal Computers (PCs) today [44, 51]. This technology is already advanced enough and is available everywhere, therefore we are able to use this technology in order to gain benefits for our end-users. Today, within our e-Society, work and education are coming closer and closer together, moreover, learning increases in importance within our information and knowledge based society. In our e-Society, the concept of Life Long Learning (LLL) is not merely a catch phrase but a necessity [17].

Whichever perspective you take into account, when talking about pervasive or ubiquitous e-Learning a complete independence of location is required which, in fact, requires more than just mobility. Even the use of notebooks cannot completely fulfill the aim of learning "wherever and whenever an end-user wants to learn" $[15,16]$. Despite the tremendous increase in educational technologies and despite the fact that the influence of these new technologies is enormous, we must never forget that learning is both a basic cognitive and social process [11].

Whilst most applications still attract the end-user's attention too much [40], we are proposing a network of interacting technologies that processes inputs and supports (via outputs) the end-users, as well as their environment, unobtrusively and ubiquitously.

On the basis of the above mentioned issues, we present three proof-of-concepts:

\section{The Ubiquitous Computing Framework (UCF)}

\section{TalkingPoints}

\section{GARFID}

Although these are only proof of concepts, due to its expendability and portability it can be seen as an experimental example for the concept of Ubiquitous Computing for developing further enhanced solutions, for example the GARFID solution to support learning of children who are either too young to use conventional input/output devices or, on the other hand, disabled or elderly people, who are again unable to manage standard input/output devices. We argue that the GARFID principle would also serve well in situations where personal computers are unsuitable, for example home-care of aged people [19], or in hospital applications ( $c f$. [18] and [46]).

\section{Example One: The Ubiquitous Computing Framework (UCF)}

The term Ubiquitous Computing (UC) has been used, in a very broad sense, to refer to a variety of

Copyright (c) 2008 John Wiley \& Sons, Ltd.

Prepared using wcmauth.cls methodologies and technologies utilizing miniature computing devices. Thus far, a plethora of frameworks have been proposed. In this paper, we emphasize the end-user-centered aspect of ubiquitous computing and have conceived a generic Ubiquitous Computer Framework (UCF).

\subsection{Pervasive, Ubiquitous, End-user-Centered}

It is interesting that Weiser $[48,51]$ followed, in his early ideas, a end-user-centered approach that uses technology only as means to an end; it supports the end-user in their daily life tasks without the technology being in the focus. The growth of the increasingly popular End-user Centered Design (UCD) approach [7, 13, 31, 43] has occurred in parallel with the focus on the end-user. UCD is an approach to creating environments and products that are usable by the end-users to the greatest extent possible [26] and to gain the maximum benefit for the end-user. Whereas System Centered Design addresses certain questions such as: What can be built on this technology? What can I create from the tools available? What do I, as a developer, find interesting to work on? - End-user Centered Design is totally based upon the end-user's abilities and needs, context, work, and tasks [12].

Through the years, many similar concepts have appeared that, on the one hand expanded the theory of UbiCom, and on the other hand basically addressed similar or identical aspects; examples are: Calm Computing [50], Invisible Computing, Hidden Computing, Ambient Intelligence [1] or Pervasive Computing [25]. The theory of Pervasive Computing (PerCom) is also about omnipresent and ubiquitous information processing and has the primary objective of usage in short-term processes [27].

The difference between these concepts, relates to the fact that UbiCom is a kind of social-philosophical approach when compared to PerCom, which is an economical-commercial utilization. Consequently, Weiser's idea should be seen as a vision and target that should be reached in the long term, but that has, as of yet, not been fully realized and should be considered a task for the future. We should emphasize that in our paper, we use the Term UbiCom synonymously with PerCom — as many other authors do ([27], [38] to mention only a few).

\subsection{Multi-disciplinarity}

The vision of UbiCom requires - as Weiser mentioned - a multidisciplinary approach which he called the

Wirel. Commun. Mob. Comput. si: 1-15 (2008) DOI: $10.1002 / \mathrm{wcm}$ 
"[...]ubiquitous computing framework [...] work impacts all areas of computer science, including hardware components (e.g. chips), network protocols, interaction substrates (e.g. software for screens and pens), applications, privacy, and computational methods" [49].

Also, Mattern assumes a collection of many IT techniques for the realization of internet related application systems, which use mobile and heterogeneous front-end devices to realize UbiCom [4, 28, 35].

Therefore we can state that UbiCom treats the question of cross-sections, whereas the integration of components and services is of main importance. This implies that for realization, all areas of hardware and software techniques, concepts, and processes have to interact with each other. The concept not only concerns hardware designers and network specialists but also software developers and specialists for human computer interaction, process engineers, and so on $[23,24,38]$.

\subsection{The UCF}

As "The real power of the concept [does not come] from any one of these devices - it emerges from the interaction of all of them" [48] mobility, dynamics and heterogeneity lead to new challenges [28]. Existing literature describes many fractional concepts of UbiCom in a very abstract form (e.g. smart spaces, smart things etc.). However, in order to integrate all currently existing concepts and aspects, the Ubiquitous Computing Framework (UCF) was designed and developed (see Figure 1). Basically, we consider the UCF as a network, consisting of many interacting technological solutions. Inputs cause actions in the network which - after some processing in the network - support, as some form of output, the end-user and his/her environment ubiquitously and unobtrusively.

The different parts of the framework are described in the following.

Input and output: The environment produces different types of input and output (I/O), which originates from individuals (humans) or stems from the natural environment (see Figure 1).

Besides classical input (including text), there are other non-classical electronic, optical, acoustic, magnetic, chemical and biometrical input channels. Newer, more natural forms of input, such as speech, gesture, facial expressions and other sensory inputs, have the potential to replace classical systems [32]. Such multimodal Human-Computer Interaction (HCI)

Copyright (c) 2008 John Wiley \& Sons, Ltd.

Prepared using wcmauth.cls systems address more than one modality (e.g., speech, gesture, handwriting, etc) and can be considered to correspond to and support human senses: cameras (sight), haptic sensors (touch), microphones (hearing), olfactory (smell), and, in future, taste [21]. Many other computer input devices activated by humans, however, can be considered to correspond to a combination of human senses, or to none at all: keyboards, mice, writing tablets, motion input (e.g., the device itself is moved for interaction), blood pressure, galvanic skin response, and other biometric sensors [41]. An example of limited interfaces is that the complexity of group interaction often hinders the performance of a whole team. Consequently, the availability of rich multimodal information and interaction about what is going on during learning and working sessions make it possible to explore the possibility of providing support to dysfunctional teams from facilitation to training sessions, addressing both the individuals and the group as a whole [33].

To interact with the environment, sensors are used, which provide the framework with values that are measured in natural environments (e.g. temperature, humidity, air-pressure, luminance, magnetism, acceleration, force etc.). Display, sound and speech are worth mentioning as output channels, as well as actors, which perform actions and are the matching counterparts to sensors.

Devices: Under some circumstances it is important to interact with the network via $\mathrm{I} / \mathrm{O}$ devices. These mainly take over I/O tasks between the end-user and the network. The device can be a PC, PDA or mobile phone, or an electronic pencil, etc. Depending on the technology used, these devices are equipped with communication units including RFID readers, GPS antennas, infrared readers, and so on. In cases where individuals interact directly with the smart object, the smart object (see next section) and the device are integrated into one single unit.

Context: To access the network, a context is needed that describes the object's background and provides end-user services depending on the context. Any communication needs a context, which acts as some kind of filter; i.e. this protects against data overload (data flooding).

Smart Objects: The core network consists of so-called smart objects, which can be smart things ${ }^{\dagger}$ as well

${ }^{\dagger}$ Smart things are small objects equipped with sensors, processing units, sensors as well as communication modules which are integrated in everyday objects. These objects can communicate with

Wirel. Commun. Mob. Comput. si: 1-15 (2008) DOI: $10.1002 / \mathrm{wcm}$ 


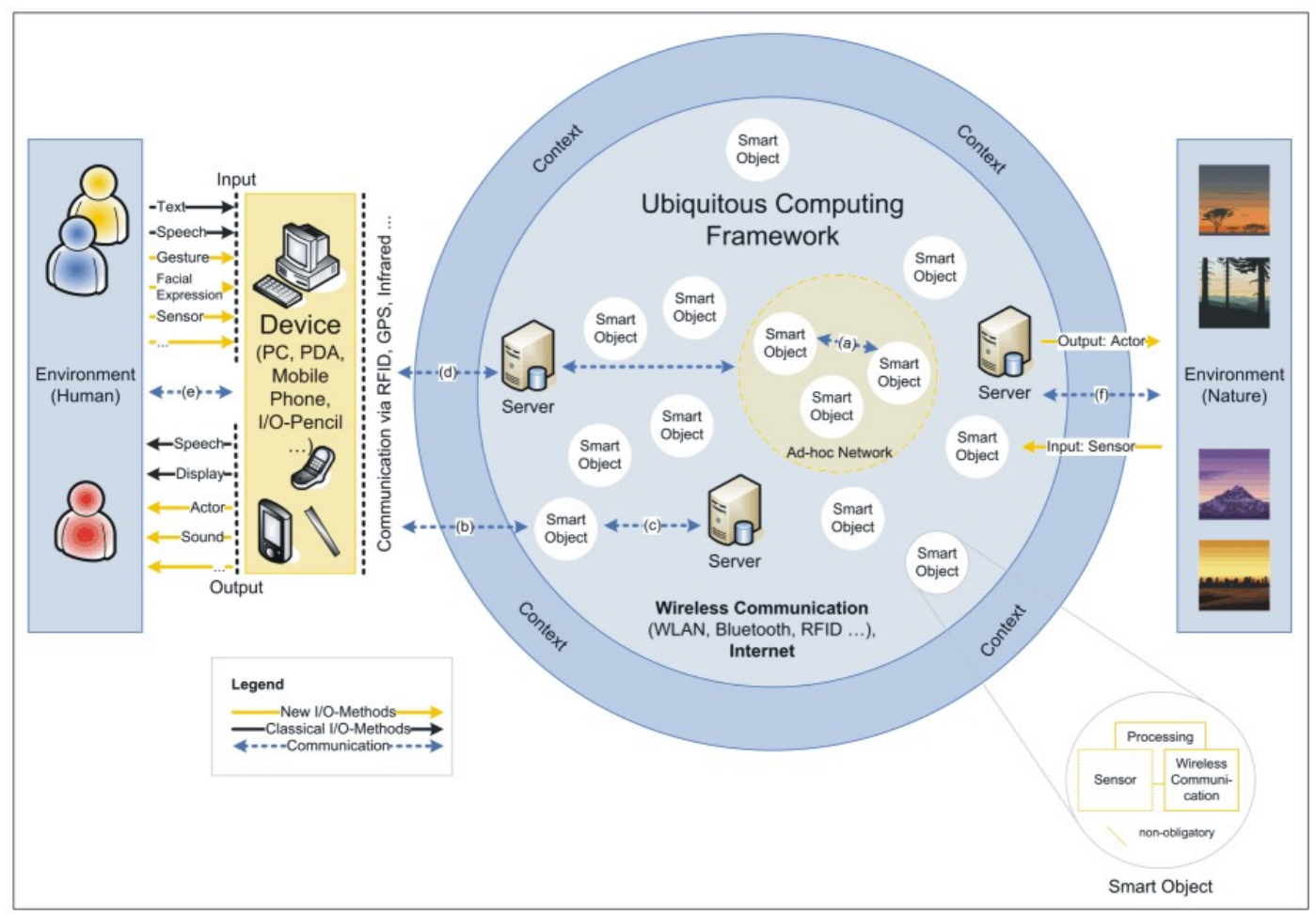

Fig. 1. Ubiquitous Computing Framework [9]

as smart spaces ${ }^{\ddagger}$. Smart objects are aware of their environment, can quasi perceive their surroundings through sensors, can collaborate with peers using short-range wireless communication technologies, and provide context-aware services to end-users in smart environments [39]. Smart objects do at least some kind of local processing of data (e.g. sending of an ID), which are sent via wireless connections to a remote server in the network. Some smart objects are additionally equipped with sensors to receive sensor values. Time and resource consuming processing can be done on remote servers, where enough capacities or energy is available. Simple I/O activities, as well as the transmission to remote locations, have to be done solely by the smart object [27]. Examples are RFID transponders, infrared beacons, and physical sensors.

Ad-hoc Network: For some applications, smart objects

each other and contact remote servers (e.g. intelligent fridge or weather-forecast-taking-in-account sprinkler).

${ }^{\ddagger}$ Smart spaces are areas where smart objects equipped with special sensors are set out to observe special ambience values. A central server bundles the data and interprets it. In this way, for examples, bushfires can be forecasted.

Copyright (C) 2008 John Wiley \& Sons, Ltd. Prepared using wcmauth.cls can form ad-hoc networks. Examples are Mobile adhoc networks (MANet), which are self-configuring and mainly work autonomously.

Internet: The internet is a very important factor for the UbiCom framework. Web services, which are used by the components of the network, must be offered (e.g. smart objects, servers).

Remote Servers: Some functions that need high processing as well as high storage capacity or large databases can be submitted to a remote device on the network (a server)

Basic I/O tasks need to be done locally - by smart objects, the rest can be done by the remote system to give the end-user the illusion that the device itself is operating the task (compare [29]). This method of storing data remotely is advantageous, since the data is not lost, even if the device mislaid.

Communication: Communication is an integral part of our concept and is mainly handled by wireless technologies, such as WLAN, Bluetooth, RFID, GPS, and infrared. During our research we found the

Wirel. Commun. Mob. Comput. si: 1-15 (2008) DOI: $10.1002 / \mathrm{wcm}$ 
following main kinds of communication, which we separated into different clusters:

1. Communication Computer-Computer:

i) Smart objects to smart objects Example: Observation of seabird nests via so-called motes [5].

ii) Smart object with device

Example: RFID reader reads from RFID tag.

iii) Smart object to server

Example: Smart objects send temperature values measured with sensors to a server that bundles this data. High temperatures could refer to a possible danger of fire, for example.

iv) Device to server

Example: A PDA with an integrated RFID reader sends a tag's ID to a web service in order to obtain information about the - to the respective tag - corresponding book.

More: device to service, server to server.

2. Communication Human-Computer:

v) Environment (human) to device Example: Mobile phone speech recognition that automatically dials the referred number, for example in an emergency.

vi) Environment (nature) to smart object

Examples: Light switches that function via radio technology could be placed in various areas of a house; temperature sensors can control the heating;

\subsection{Enabling technologies of UCF}

There are two specifics that should be mentioned in order to make Section 3.2 more understandable: the so-called data shadow and the context.

UbiCom produces a lot of data, which can be merged in a model and presented as a digital image of the real world. These digital world models form the basis for context oriented applications [37].

Remote identification is an important characteristic of smart objects [27]. Due to identification technologies - such as RFID, infrared and GPS - objects can be identified unmistakably. The corresponding dataset is found in a database on the internet. This set of data the data shadow - is the external memory of the object;

Copyright (C) 2008 John Wiley \& Sons, Ltd.

Prepared using wcmauth.cls all the object associated information is stored in some kind of virtual storage (database).

As UbiCom is a general concept, it combines many technologies. To realize its application, different techniques can be used, e.g. WLAN, Bluetooth, infrared, and RFID. As this work utilizes RFID a short insight follows: RFID stands for Radio Frequency Identification and is a radio technology, which allows reading and writing data without physical contact.

Its most important elements are:

- reader: the sending and receiving unit that reads and eventually stores data on one or more servers

- transponder (or tag): which contains data. Tags are differentiable in that some are read-only (they only store a unique ID), while others are readable and writable.

Both the reader and transponder are equipped with an antenna (dipole antenna or coil) to send and receive signals.

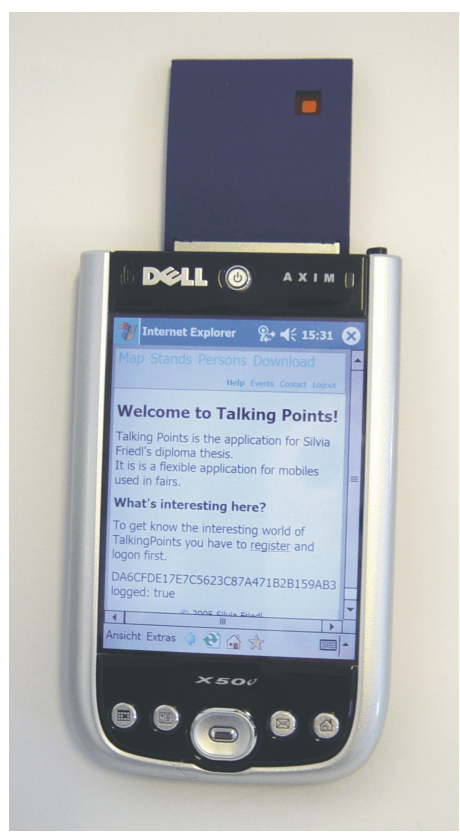

Fig. 2. PDA with RFID reader

RFID is generally seen as a comprehensive technical infrastructure. Therefore, this simple system should be extended by another component: integrated systems. Integrated systems are servers and applications that are connected to the reader.

Wirel. Commun. Mob. Comput. si: 1-15 (2008) DOI: $10.1002 / \mathrm{wcm}$ 


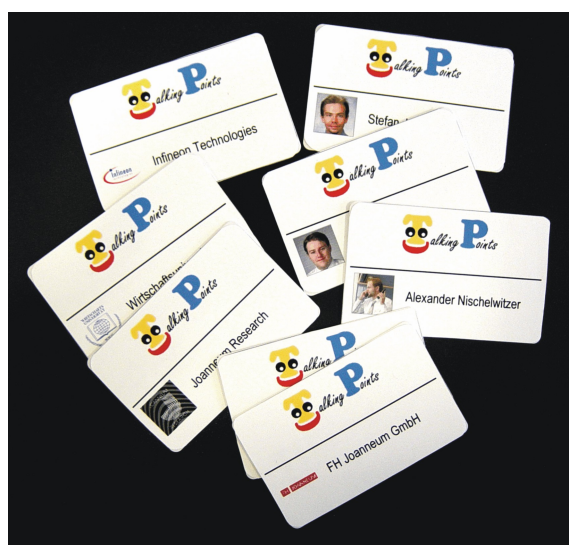

Fig. 3. $13.56 \mathrm{MHz}$ Transponder

A context is the information which describes the situation of an entity, which can be a person but also could be a location or object [37]. To use applications ubiquitously the context of a end-user must be defined adequately.

Some definitions for the context are:

Situation: (a) Demographic Data (age, language, education): authentication [45] can adapt the viewable features. (b) Optimization of Display View: optimized to the corresponding device (e.g. PC, PDA, mobile phone).

Location: the end-user's current location and surroundings, sent via RFID GPS or infrared.

Activity: the end-user's activity, e.g. recognition via wearable computing devices, mobile phone

Context-aware systems use contextual information to adapt the system and its behavior to the end-user. Additionally, they may also try to make assumptions about the end-user's current situation. Example: A context-aware mobile phone may know that it is currently in a meeting room, and that the end-user is currently in a meeting. It could then either suppress or reject calls deemed unimportant. In such systems, novel solutions for end-user-centric service discovery are crucial in providing personalized views of only the services of potential interest, which is based on factors such as the end-user context, end-user preferences, the access device's capabilities, and environmental conditions [2].

Consequently, the context can influence an application's behaviour [37]:

Copyright (c) 2008 John Wiley \& Sons, Ltd. Prepared using wcmauth.cls
1. Context based Selection: Services and Information are offered depending on the context.

2. Context based Presentation: Presentation is based on the context (e.g. different output channels are used: audio, video, text)

3. Context based behavior: Actions are initiated in regard to the context (e.g. depending on the device's location or the current situation calls are forwarded to another phone number).

To benefit from the potential of UbiCom, a combination of data shadow and context is needed. This combination enables a realization of Weiser's vision [48], in order to ubiquitously support an individual's everyday tasks.

Due to the context and the remotely fetched data shadow, the end-user gets the impression that the tasks are made by the device itself. That the information is sourced from the internet is not obvious or noticeable to the end-user [27].

Example: The end-user manual of the television, the recipe of a microwavable meal, or the package insert of medicine could all be obtained up-to-date from the internet.

\section{Example Two: TalkingPoints I}

The original idea was to use TalkingPoints for trade fairs, however, while experimenting with it, we identified a variety of possible applications, including e-Learning.

\subsection{Motivation and Objectives}

There were two requirements when our work began: the application should be mobile, and should use RFID technology [22]. Trade fairs were chosen because a lot of interaction (person-person, person-product) (place, products, people, etc.) occurs and a lot of information is displayed at the same time.

Target Group and its Needs: The audiences of fairs are people who strongly depend on their personal contact networks. Therefore they want to use their contact data everywhere, e.g. on their notebook or PDA.

Problem: When attending such fairs, many people are introduced to each other and more and more contact data is collected. These data, in most cases, is available in loose paper form. To use this information everywhere, it has to digitized, which is very time consuming. Therefore in most cases it is not done. As

Wirel. Commun. Mob. Comput. si: 1-15 (2008) DOI: $10.1002 / \mathrm{wcm}$ 
a consequence, the information is available but, due to not being in digitalized form, its use is limited.

Solution: The concept of TalkingPoints allows the system to collect contact information ubiquitously during a fair and download it in an adequate form afterwards. Additionally the end-user can obtain useful information about objects during their visit; For deeper personalization of the system, the end-user can add some notes to the object which are also available as download.

Benefit: As contact data is available without media discontinuity, it is used in its entirety and personal jobrelated networks can be used optimally. Additionally, each stand's information and website can be displayed, as can each person be "RFIDentified".

\subsection{TalkingPoints Technology}

TalkingPoints is dealing with two different objects: stands (where each stand is represented by a company) and visitors.

At first, specific information about every object must be provided, e.g. e-mail, name, and homepage. Every object is equipped with a transponder, which uniquely identifies the object. Other possible objects could be paintings or sculptures in museums, sights for sight-seeing tours, and so on.

Every end-user obtains a mobile device (e.g. a PDA) with an integrated RFID reader. If there is a fair object in the reading range of the reader, the object's UID is read. This UID is sent to a server in the internet, which returns the corresponding object site (indirect sensing, $c f .[22])^{\S}$.

If there is a physical invocation of sites - as mentioned above - this is called automatical/physical hyperlinks (push ${ }^{\top}$ ) compared to manual/virtual hyperlinks, where the end-user themself requests the site $\left(\right.$ Pull $\left.^{\|}\right)$. For the second kind of links, the end-user need not to be at the location of interest as they are able to see object information in advance. Also RFID security with secure tags can be used [46][47].

During the fair all participants can collect information about all fair objects to obtain a personal catalogue and contact list. These lists are provided in different formats during and after usage.

\footnotetext{
$\S$ As the objects tell information about themselves the application is called TalkingPoints.

I Push: Contents are provided without end-user interaction.

\| Pull: Contents must be requested by the end-user.

Copyright (c) 2008 John Wiley \& Sons, Ltd.

Prepared using wcmauth.cls
}

\subsection{Design and Development}

At the beginning, the application was defined by the objectives and the motivation. Thereafter an analysis of competitive applications was done. Different scientists developed similar applications; all used the following simplified pattern: An end-user equipped with a handheld device automatically obtains information about objects in their near environment [22, $30,45]$. Insights of these applications incorporate TalkingPoints and its detailed design. Later, use cases were defined to simplify the programming.

Resources: The work used technologies that are commonly used and accepted and therefore affordable. The following resources have been used.

- PDA: Dell Axim X50v (Pocket PC 2003 operating system) with integrated Compact Flash II interface to use an ACG RFID handheld reader and WLAN interface.

- Transponders: $13.56 \mathrm{MHz}$ tags (ISO 15693) from Tagnology.

- WLAN: Access to the local WLAN necessary to use the internet and handle the communication with the web server.

- Web server: an Apache Tomcat/5.5.7 web server with a MySQL Version 4.1.10a database and JSP.

Local Application: A local application is used to read the UID from the transponder and to manage the PDA's reader, as well as to offer an interface for login and registration functions. The application runs in the background and regularly polls the reader interface as to whether there is a tag within reading distance. When one is found, the transponders UID is read and, depending on the user's context (Register, Login and Show object), specific parameters are sent to the web server which returns the corresponding site. Therefore, the mobile usability is improved and the user can work with the applications without real navigation and hands-on interaction.

Web application: This part offers the main functionalities. Data is stored in a database to provide more data security in case of a crash or loss of the PDA device. All use cases use the same web application. With the use of different style sheets, it is possible to adapt the display output according to the output device (PDA or PC). Essentially, there is the usage of div (divisions), which permits areas of the HTML site to be grouped and to place and format them separately.

Wirel. Commun. Mob. Comput. si: 1-15 (2008) DOI: $10.1002 / \mathrm{wcm}$ 


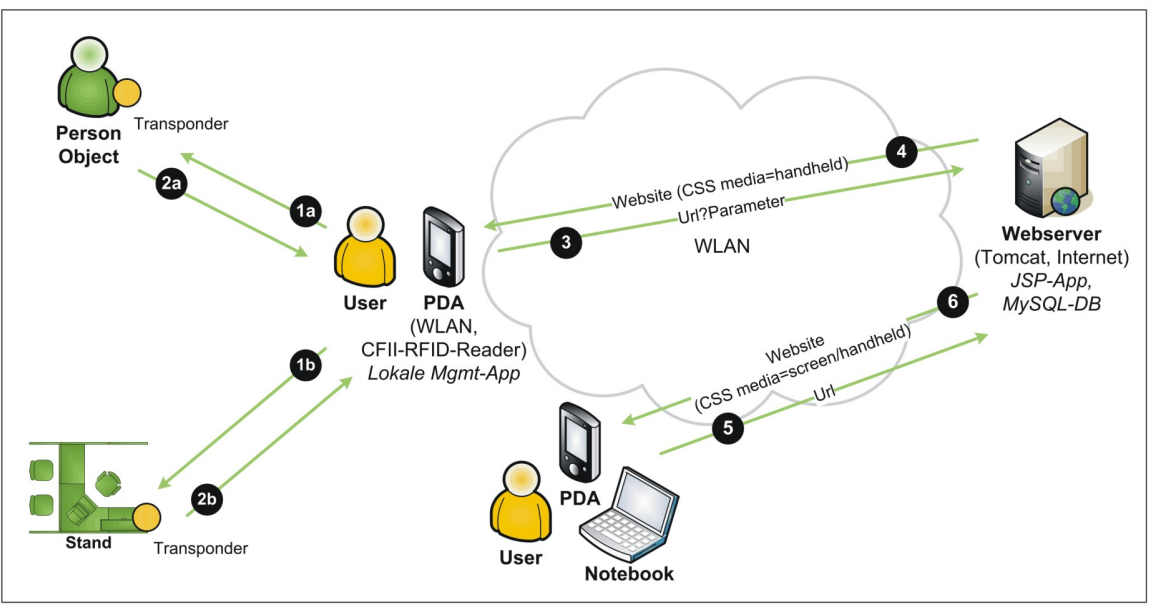

Fig. 4. Technologies used in TalkingPoints

Therefore, the structure for every client is the same and only the output is adapted to the special needs of the separate browsers (compare Figure 7 and Figure 8). The web server offers a central point for requests from a PDA, which interprets the incoming parameters and refers the user to the matching site. This process is called indirect sensing as the user does not request the corresponding site directly.

\subsection{Parallels of TalkingPoints compared to UCF}

The following section points out the connections between the UbiCom framework and the TalkingPoints solution.

Data Shadow: The solution shows a representative usage of the data shadow concept.

Context: For practical use, the context of identity and location (RFID identification and data mapping) is important. The use of context based selection (some functions are only available when logged in) and context based presentation (different views depending on the device) characterize the solution.

Physical and Virtual Links: The application uses both types of links: physically/automatically set links so the user can move in the real world and virtual/manual links so that he/she can also receive information virtually.

Internet of Things: As the application is mainly realized for the web browser and only a small part takes over RFID tasks (compare section 3.3), we can

Copyright (c) 2008 John Wiley \& Sons, Ltd.

Prepared using wcmauth.cls see parallels to smart objects (more precisely: smart things) as mentioned in UbiCom.

The reason for using a web-based solution is obvious: processing-intensive activities are done on the web server and small devices such as the PDA just complete I/O tasks.

\subsection{Functionalities}

Depending on the current status of the user, different functions are offered. We distinguish between three states: (a) Permanent, (b) Logged on and (c) Logged off. Some functions are offered permanently, such as contact and home. In addition to the permanent functions, there are some functions only available when the user is logged on or logged off. Functions that are available when the user is logged off are: the password reminder, register and login.

The following functions, among others, are the core functions, which are only available if the user is logged on.

- Graphical Map

- List of Stands

- List of Persons

- Object's Details

- Download of Object's Data

List of Stands: This function shows a list of all stands available at the fair. The stands are arranged after their states and names. States could be: current stand (red), visited stand (blue) and not yet visited stands (white).

Wirel. Commun. Mob. Comput. si: 1-15 (2008) DOI: $10.1002 / \mathrm{wcm}$ 


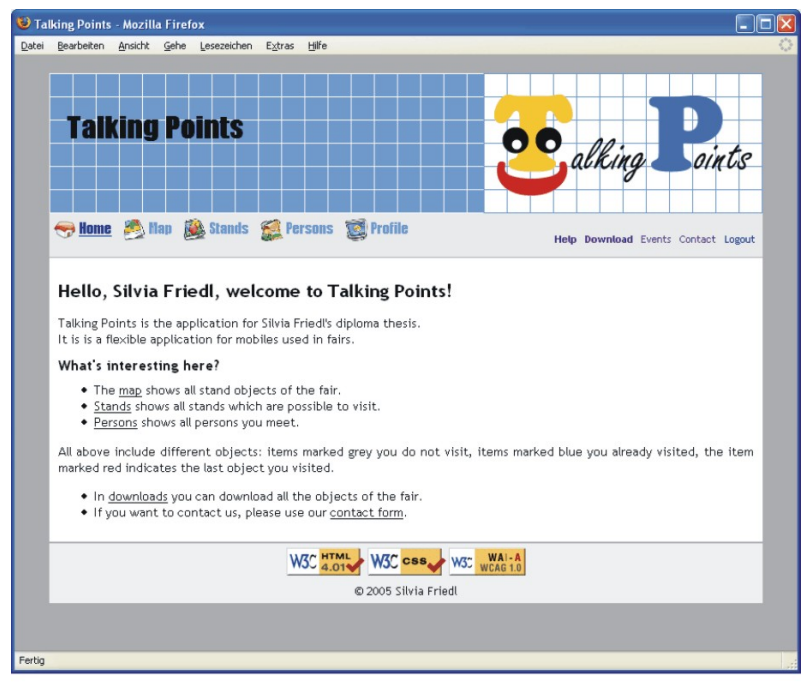

Fig. 8. Start page on the PC

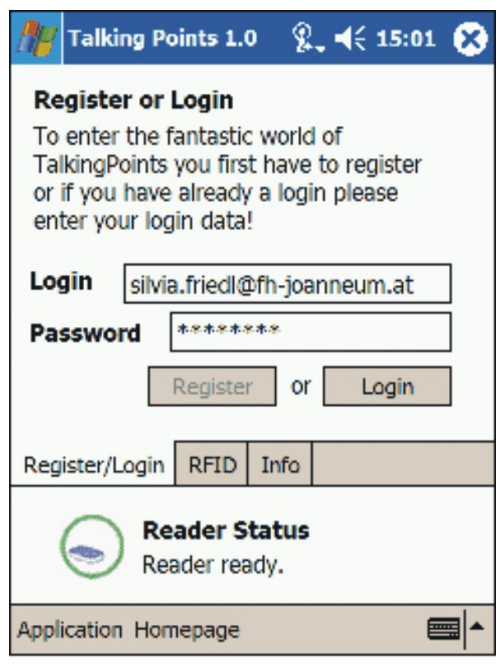

Fig. 5. Function "Register" and "Login"

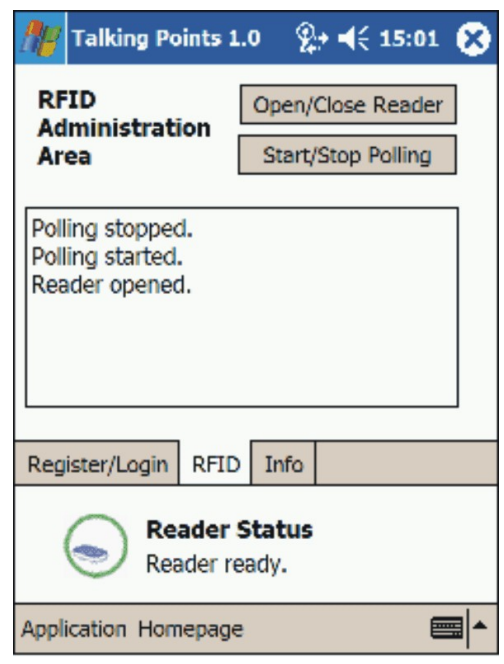

Fig. 6. Function "RFID reader"
When clicking the stand's name the stand's details are shown (see Figure 15).

List of People: Likewise a list of all persons attending the fair can be shown. Two states are possible: blue, if the user has already met that person and grey, if the user has not yet met that person. When clicking the person's name the person's details are shown (see Figure 16).

Graphical Map (Map): The map shows the location of

Copyright (c) 2008 John Wiley \& Sons, Ltd.

Prepared using wcmauth.cls all stands at the fair. Every stand indicates the name of the company and the location of the transponder at the stand.

Object's Details: Since objects can be persons, as well as stands, different object views are available (see Figure 15 and Figure 16). The details can not be selected from the menu; there are the following possibilities of choosing:

Wirel. Commun. Mob. Comput. si: 1-15 (2008) DOI: $10.1002 / \mathrm{wcm}$ 


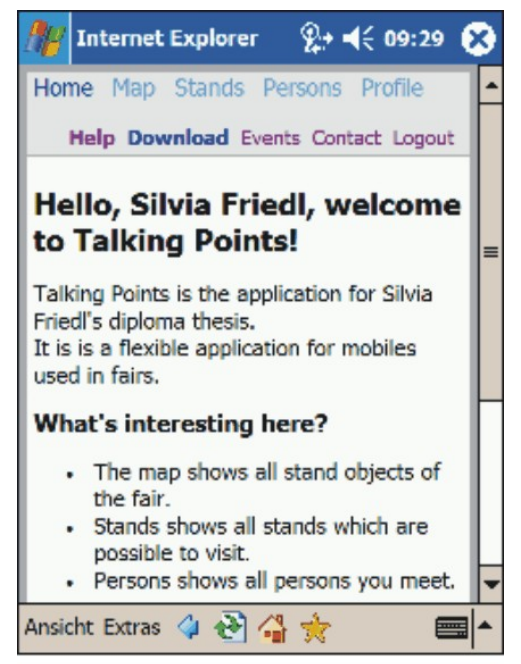

Fig. 7. Start page on the PDA

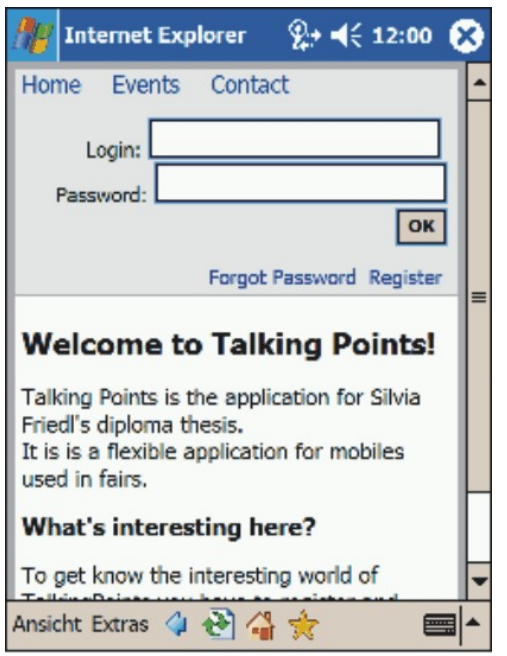

Fig. 9. Functions if status logged off and permanent

(a) Automatic/physical hyperlink: A view of the details is initiated by the local PDA program.

(b) Manual/virtual hyperlinks: Details can be chosen in the browser by clicking an object (on the graphical map or the object list for stands or persons).

In the objects view, the functions recommender, notes and "add a contact" are available. The recommender suggests objects on the basis of existing user data and is only realized for stands. Every user

Copyright (c) 2008 John Wiley \& Sons, Ltd. Prepared using wcmauth.cls

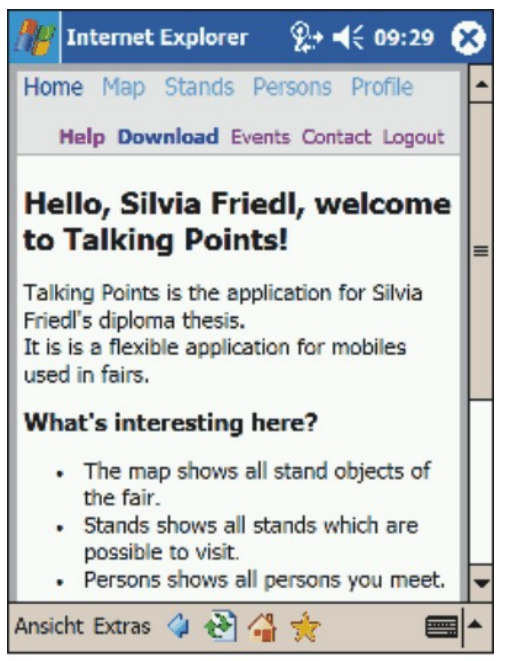

Fig. 10. Functions if status logged on and permanent

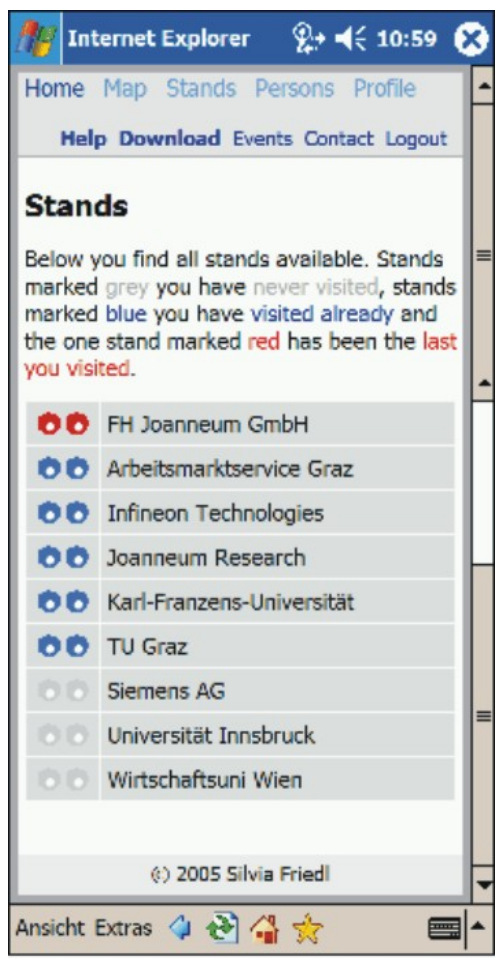

Fig. 11. List of Stands

can add some notes to every object which is stored on the user basis. These notes can be downloaded afterwards via the download function. Objects can be

Wirel. Commun. Mob. Comput. si: 1-15 (2008) DOI: $10.1002 / \mathrm{wcm}$ 


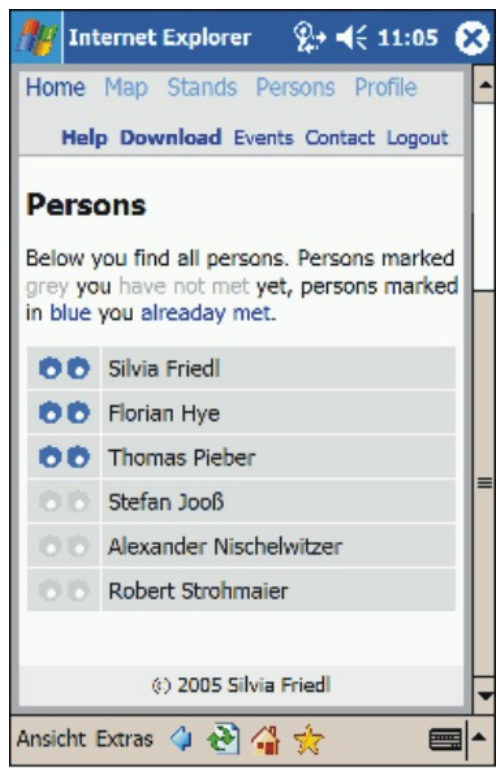

Fig. 12. List of People

selectively added to the PDA's local contacts by using the add contact function, which uses Virtual Card File (VCF) files.

Downloading of Object's Data (Download) is performed as follows: During and after the usage of TalkingPoints, personalized lists can be created. The lists are available in different formats: HTML, CSV and VCF. The CSV file can be used for the import of contacts into mail programs, the VCF files for an import of the same on to the PDA. The user also has to decide whether they want to download the visited, not visited or all stands as well as the met or not met persons for the later created list**.

\subsection{Discussion}

TalkingPoints provides a ready platform upon which other scenarios can be evaluated and validated. In the case of e-learning, a personalised curriculum is important. It can be envisaged that by embedding RFID transponders into personal identification cards, e.g. staff/student ID, upon walking into a lecture room equipped with RFID readers, an individual's PDA or Smartphone will be connected to the backend systems to pull out or update the relevant information, e.g. open questions they have regarding previous courses, the

** Downloads of not met persons are excluded.

Copyright (C) 2008 John Wiley \& Sons, Ltd.

Prepared using wcmauth.cls progress so far, and the status of previously studied models. Some of these data might be sent via RFID directly, if the amount of information is limited; others might be transferred through another type of connection (e.g. Bluetooth or WiFi) subsequently established after the initial exchange of information via RFID. The advantage is evident, without being aware of the communication with the environment; one is taken through the necessary negotiation procedure to acquire the most appropriate learning materials. The learning site can be further extended to less conventional places, e.g. office room, cafeteria, etc., in order to distribute and better locate the resources.

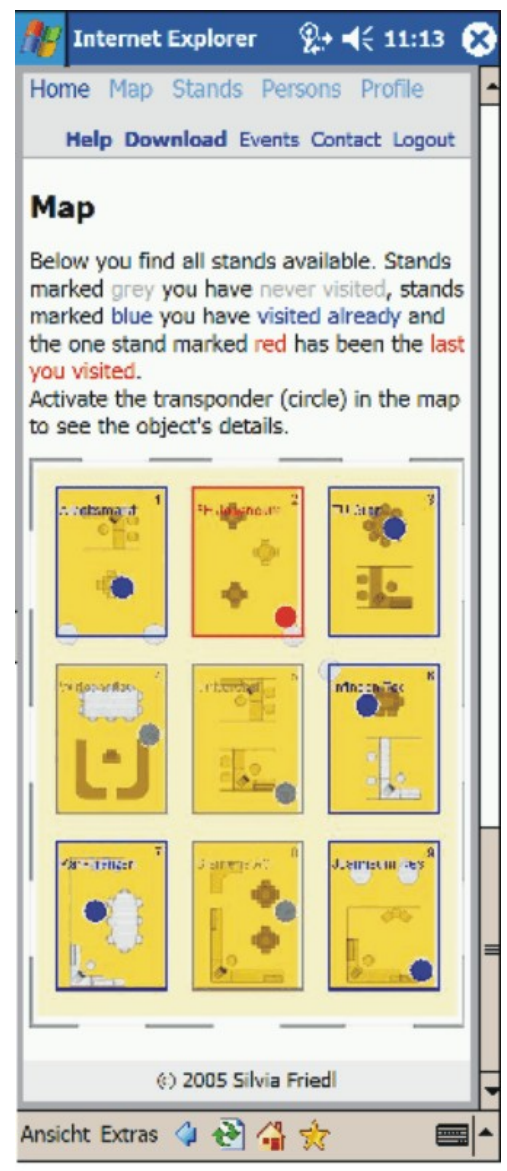

Fig. 13. Graphical Fair Map

Wirel. Commun. Mob. Comput. si: 1-15 (2008) DOI: $10.1002 / \mathrm{wcm}$ 


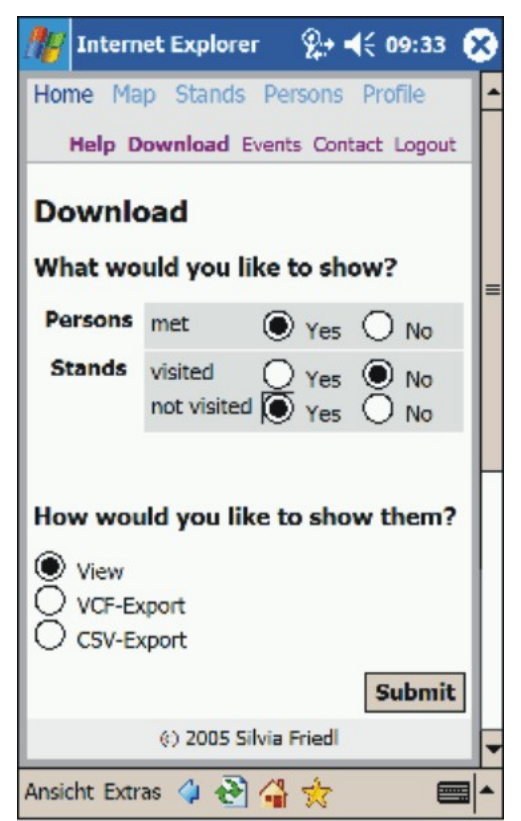

Fig. 14. Download Area

Fig. 15. Stand Object

Copyright (C) 2008 John Wiley \& Sons, Ltd. Prepared using wcmauth.cls

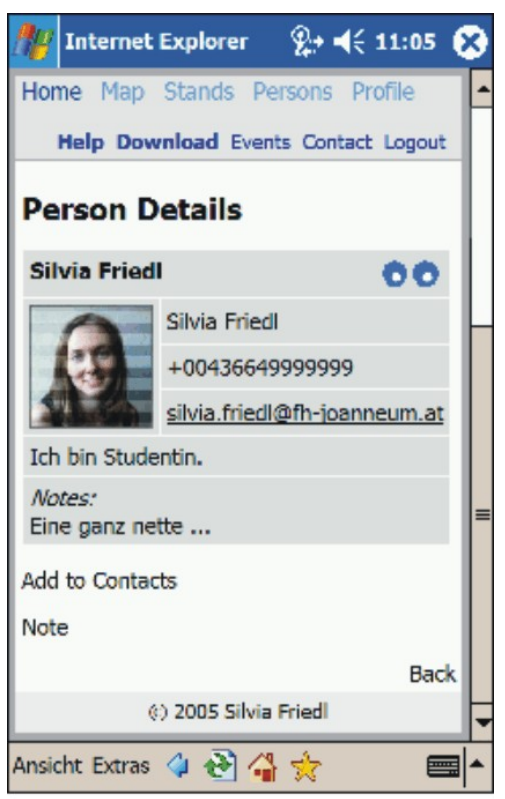

Fig. 16. Person Object

\section{Example Three as a future outlook: GARFID}

With TalkingPoints, we were able to demonstrate the benefit and added value achieved through "tagging" human objects with RFID transponders to allow information sharing. However, in practice, the use of RFID technology can be much more versatile. Instead of "tagging" human beings, RFID transponders can be embedded into learning materials in order to build a network of "smart" and context-aware learning objects upon which ubiquitous learning environments can be laid. GARFID can be deemed as the first step towards exploring the potential along this track of research.

\subsection{E-Learning for the youngest}

Environments that provide learners opportunities to apply their previous knowledge appropriately [14] and invite exploration can lead to higher levels of motivation and interest; unfortunately, such learning strategies are still rarely used today because they are difficult to implement [6].

In 1999, IBM started their early childhood education programs for children aged 3-6 years. The goal was an introduction of information and communications technology (ICT) in preschool. In fact, this is supported by IBM's KidSmart

Wirel. Commun. Mob. Comput. si: 1-15 (2008) DOI: $10.1002 / \mathrm{wcm}$ 
Early Learning Program, which includes specifically designed computer learning centers and special software.

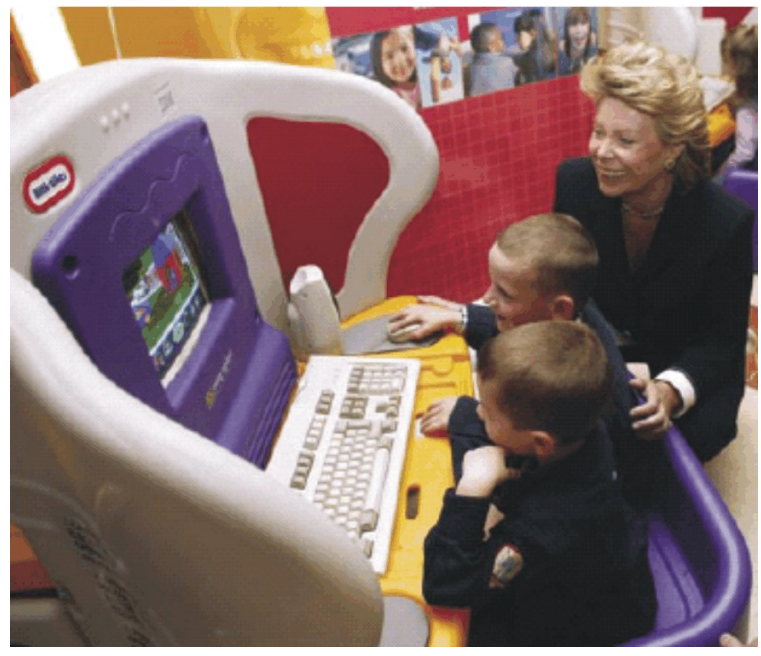

Fig. 17. IBM’s KidSmart [20]

Learning for children should be more than just the use of a personal computer. At the same time, they can train their social skills, behaviour, thinking skills, concentration, memory, and language development [36]. This means that the creativity and innovation ability needs to be assisted by the whole abundance of Information Technology, including programmable toys, floor robots, digital cameras, scanners, tape recorders, video recorders and of course mobile phones. The two main goals include: Computers and technology should not limit or hinder the creativity of children; and the notion of play is at the centre of young children's focus [20].

\subsection{Motivation and Objectives}

Why is new technology only considered to be a method of making stressed business men's lives more comfortable? Small children are also able to use - and benefit from - new technology.

Target Group and its Needs: The system we developed was especially designed for children aged 3-6 years. However, even children above or below that age can have fun playing some of the games. The degree of difficulty can be adapted to the age as necessary. Actually, play has a large potential for learning $[8,34$, 42].

The only requirements to work with KidSmart are a computer and the corresponding software package.

Copyright (C) 2008 John Wiley \& Sons, Ltd.

Prepared using wcmauth.cls
The special design of the learning center is just to get the children's attention.

Problem: Children have to use the standard input devices that are known from PCs. On the one hand this could be an advantage because children learn to use them at a very early age, but on the other hand this could become a big problem, especially for the youngest ones - at the age of three and below. Exactly here, multimodal interfaces can become a big challenge: first to read the right letters and find them on the keyboard and it will be hard to show them how to move the mouse in the right direction.

Solution: Maybe the use of RFID could be the solution for special input devices. Adding a tag to a toy enables the computer system to recognize this object and give a special designed respond.

Benefit: Even babies are able to interact with such technological gadgets after their first year. It is only necessary to position their toys by the RFID reader. Of course, the output of the computer depends on the children's age. For example, the name of the object could be spoken out loudly. To a certain extent, this can help alleviate the task of answering the same questions again and again.

\subsection{GARFID in a Nutshell}

GARFID is an idea to teach young children important things of life. In fact many software solutions for elearning already exist. However, the big problem is that in most cases a personal computer is necessary and the software needs to be installed first. Generally, two-year-old children and sometimes older people are not able to solve this task.

GARFID is a small mobile device packed in a childproof box. In the beginning, the objects have to be labeled with RFID tags and GARFID has to be trained with output sequences whenever there is not a ready trained set of objects delivered with the device. Even a wireless connection to the Internet is imaginable.

\subsection{Design and Realization}

GARFID can be seen as an extension and improvement to actual available E-Learning tools, we are not proposing a completely new development-it is just a model: The components which are needed are the mobile device and a transponder for each object. These contain a serial number which can be associated to the object. A wireless connection can also be used to expand the system and a notebook can be useful to evaluate the learning progress. 


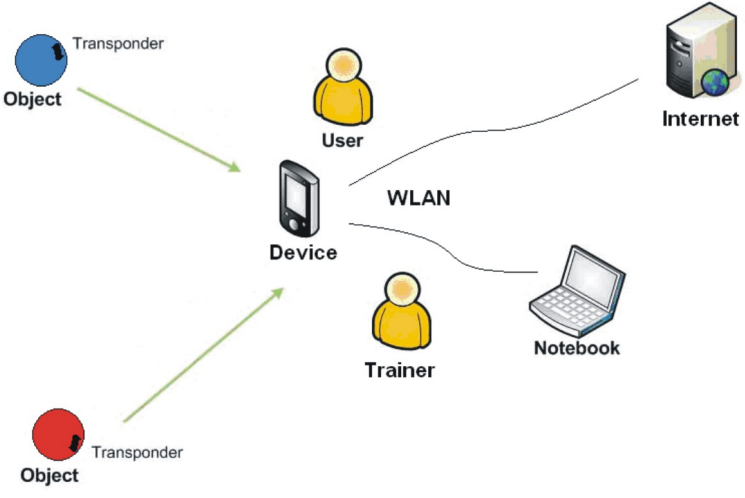

Fig. 18. Technological overview of GARFID

\subsection{Functionalities}

Once GARFID is trained, it is only necessary to put an object within the coverage of the device. The serial number is transmitted and the device reads out the name of the object. This can be done in a row, always teaching new meanings.

A sample dialog could be the following:

- The blue ball is put to the device.

- $\Rightarrow$ "This is a ball."

- If the ball is shown again.

- $\Rightarrow$ "The ball is blue."

- Etc.

Of course this can also be used for other purposes, for example to teach foreign languages, i.e. "This is a blue ball" in German "Dies ist ein blauer Ball."

\section{Conclusion and Future Work}

Although countless solutions using RFID exist in the area of UbiCom, this paper shows a scope for a new application which generates additional value. Moreover, some potential for expanding the application was found.

We presented TalkingPoints, which, although it is only a proof of concept, can be of much value for future experiments due to its expandability and portability. It should be seen as an important basic example for the concept of UbiCom for further enhanced solutions and studies. Due to its flexible definition, TalkingPoints is not only applicable in the area of trade fairs; by making some adaptations, it can be used in many areas such as museums, outdoor areas such as parks or cities as well as in the medical

Copyright (C) 2008 John Wiley \& Sons, Ltd. Prepared using wcmauth.cls field - and consequently support the idea of Life Long Learning. Additionally, it could be shown that we are on the way towards reaching the vision of UbiCom. Although Weiser's vision was claimed years earlier, it is becoming - with an increasing amount of new applications - more and more true. Based on the experience gathered with TalkingPoints, we see a big functionality for GARFID and therefore a wide application area. For example, the therapeutic aspect, even for older or handicapped people, should be mentioned. There is still much work to do in this vast area of research and, most of all, it should be done towards making life easier for humans and support learning and working in daily activities. Consequently, the bridge between Psychology and Computer Science is a necessity in the design and development of enduser centered applications.

\section{References}

1. E. Aarts. Ambient intelligence: A multimedia perspective. IEEE Multimedia, 11(1):12-19, 2004.

2. P. Bellavista, A. Corradi, R. Montanari, and A. Toninelli. Context-aware semantic discovery for next generation mobile systems. IEEE Communications Magazine, 44(9):62-71, 2006.

3. J. Bohn. Prototypical implementation of location-aware services based on a middleware architecture for superdistributed RFID tag infrastructures. Personal and Ubiquitous Computing, 12(2):155-166, 2008.

4. J. Bohn and F. Mattern. Super-Distributed RFID Tag Infrastructures. In P. Markopoulos, B. Eggen, E. Aarts, and J. Crowley, editors, Proc. 2nd European Symposium on Ambient Intelligence (EUSAI 2004), number 3295 in LNCS, pages 1-12. Springer-Verlag, Nov. 2004.

5. D. E. Culler and H. Mulder. Smart sensors to network the world. Scientific American, 7:52-59, 2004.

6. A. v. Dam, S. Becker, and R. M. Simpson. Next-generation educational software: why we need it and a research agenda for getting it. In ACM SIGGRAPH 2007, pages 26-43, 2007.

7. S. Dray and D. Siegel. User-centered design and the "vision thing". Interactions, 5(2):16-20, 1998.

8. M. Ebner and A. Holzinger. Successful implementation of user-centered game based learning in higher education - an example from civil engineering. Computers \& Education, 49(3):873-890, 2007.

9. S. Friedl. Talkingpoints - mobile rfid-anwendung im umfeld des ubiquitous computing. Master's thesis, Digital Media Technologies. Graz, FH Joanneum GmbH, University for applied Science, 2005.

10. H. Gellersen. Smart-Its: computers for artifacts in the physical world. Communications of the ACM, 48(3):66, 2005.

11. A. Holzinger. Multimedia basics, volume 2: Learning, 2002.

12. A. Holzinger. Usability engineering for software developers. Communications of the ACM, 48(1):71-74, 2005.

13. A. Holzinger and M. Errath. Mobile computer webapplication design in medicine: some research based guidelines. Universal Access in the Information Society International Journal, 6(1):31-41, 2007.

14. A. Holzinger, M. Kickmeier-Rust, and D. Albert. Dynamic media in computer science education; content complexity and learning performance: Is less more? Educational Technology \& Society, 11(1):279-290, 2008.

Wirel. Commun. Mob. Comput. si: 1-15 (2008) DOI: $10.1002 / \mathrm{wcm}$ 
15. A. Holzinger, A. Nischelwitzer, and M. Meisenberger. Lifelong-learning support by m-learning: Example scenarios. ACM eLearn Magazine, 2005.

16. A. Holzinger, A. Nischelwitzer, and M. Meisenberger. Mobile phones as a challenge for m-learning: Examples for mobile interactive learning objects (milos). In Proceedings of the $3 \mathrm{rd}$ IEEE PerCom, pages 307-311. IEEE Press, 2005.

17. A. Holzinger, A. K. Nischelwitzer, and M. D. KickmeierRust. Pervasive e-education supports life long learning: Some examples of $\mathrm{x}$-media learning objects, 2006. Online available: http://www.wccee2006.org/papers/445.pdf, last access: 200706-24.

18. A. Holzinger, K. Schwaberger, and M. Weitlaner. Ubiquitous computing for hospital applications: Rfid-applications to enable research in real-life environments. In COMPSAC '05: Proceedings of the 29th Annual International Computer Software and Applications Conference (COMPSAC'05) Volume 2, pages 19-20. IEEE Computer Society, 2005.

19. A. Holzinger, G. Searle, and A. Nischelwitzer. On some aspects of improving mobile applications for the elderly. In HCI (5), pages 923-932, 2007.

20. IBM. Early learning in the knowledge society: Report on an European conference, 2003. Online available: http://www.ibm.com/ibm/ibmgives/downloads/early_learning.pdf, last access: 2005-11-02

21. A. Jaimes and N. Sebe. Multimodal human-computer interaction: A survey. Computer Vision and Image Understanding, 108(1-2):116-134, 2007.

22. T. Kindberg, J. Barton, J. Morgan, G. Becker, D. Caswell, P. Debaty, G. Gopal, M. Frid, V. Krishnan, H. Morris, J. Schettino, B. Serra, and M. Spasojevic. People, places, things: web presence for the real world. Mob. Netw. Appl., 7(5):365-376, October 2002

23. J. Kjeldskov and M. B. Skov. Exploring context-awareness for ubiquitous computing in the healthcare domain. Personal and Ubiquitous Computing, 11(7):549-562, 2007.

24. J. Lai, S. Mitchell, M. Viveros, D. Wood, and K. M. Lee. Ubiquitous access to unified messaging: A study of usability and the use of pervasive computing. International Journal of Human-Computer Interaction, 14(3-4):385-404, 2002.

25. K. C. K. Lee, W. C. Lee, and S. Madria. Pervasive data access in wireless and mobile computing environments. Wireless Communications \& Mobile Computing, 8(1):25-44, 2008.

26. R. Mace, G. Hardie, and J. Place. Accessible environments:toward universal design. In W. Preiser, J. Vischer, and E. White, editors, Design interventions: Towards a more human architecture, page 44. New York, Van Nostrand Reinhold, 1991

27. F. Mattern. Vom verschwinden des computers - die vision des ubiquitous computing. In F. Mattern, editor, Total vernetzt, pages 1-41. Springer-Verlag, Apr. 2003.

28. F. Mattern. Pervasive / ubiquitous computing. InformatikSpektrum, 24(3):145-147, June 2004.

29. F. Mattern. Ubiquitous computing: Szenarien einer informatisierten welt. In A. Zerdick, A. Picot, K. Schrape, J.-C. Burgelman, R. Silverstone, V. Feldmann, D. Heger, and C. Wolff, editors, E-Merging Media - Kommunikation und Medienwirtschaft der Zukunft, pages 155-174. SpringerVerlag, 2004.

30. A. Nischelwitzer, A. Holzinger, and M. Meisenberger. Usability and user-centered development (ucd) for smart phones - the mobile learning engine (mle) a user centered development approach for a rich content application. In Proceedings of Human Computer Interaction International (HCII), 2005. Lawrence Erlbaum Associates.

31. D. Norman and S. Draper. User Centered System Design. Hillsdale (NY), Erlbaum, 1986.

32. S. Oviatt, T. Darrell, and M. Flickner. Multimodal interfaces that flex, adapt, and persist. Communications of the ACM,
47(1), 2004.

33. F. Pianesi, M. Zancanaro, E. Not, C. Leonardi, V. Falcon, and B. Lepri. Multimodal support to group dynamics. Personal and Ubiquitous Computing, 12(3):181-195, 2008.

34. J. Robertson and C. Howells. Computer game design Opportunities for successful learning. Computers \& Education, 50(2):559-578, 2008.

35. K. Romer and F. Mattern. The design space of wireless sensor networks. IEEE Wireless Communications, 11(6):54 61, 2004.

36. J. M. Roschelle, R. D. Pea, C. M. Hoadley, D. N. Gordin, and B. M. Means. Changing how and what children learn in school with computer-based technologies. Future of Children, 10(2):76-101, 2000.

37. K. Rothermel, M. Bauer, and C. Becker. Digitale Weltmodelle - grundlage kontextbezogener systeme, 2003.

38. M. Satyanarayanan. Fundamental challenges in mobile computing. In PODC '96: Proceedings of the fifteenth annual ACM symposium on Principles of distributed computing, pages 1-7. ACM, 1996.

39. F. Siegemund, C. Floerkemeier, and H. Vogt. The value of handhelds in smart environments. Personal and Ubiquitous Computing, 9(2):69-80, 2005.

40. W. S.M., J. Patrick, P. Morgan, and S. King. Influencing cognitive strategy by manipulating information access. Computer Journal, 50(6):694-702, 2007.

41. C. Stickel, J. Fink, and A. Holzinger. Enhancing universal access - EEG based learnability assessment. In C. Stephanidis, editor, Universal Access to Applications and Services, pages 813-822. Berlin, Heidelberg, New York, Springer, 2007.

42. L. Terrenghi, M. Kranz, P. Holleis, and A. Schmidt. A cube to learn: a tangible user interface for the design of a learning appliance. Personal and Ubiquitous Computing, 10(2-3):153158, 2006.

43. K. Vredenburg, J.-Y. Mao, P. Smith, and T. Carey. A survey of user-centered design practice. In CHI '02: Proceedings of the SIGCHI conference on Human factors in computing systems, pages 471-478. ACM, 2002.

44. R. Want. Rfid - a key to automating everything. Scientific American, 290(1):56-65, 2004.

45. R. Want, K. Fishkin, A. Gujar, and B. Harrison. Bridging physical and virtual worlds with electronic tags. In $\mathrm{CHI}$ '99: Proceedings of the SIGCHI conference on Human factors in computing systems, pages 370-377. ACM, 1999.

46. E. Weippl, A. Holzinger, and A. M. Tjoa. Security aspects of ubiquitous computing in health care. Springer Elektrotechnik \& Informationstechnik, e\&i, 123(4):156-162, 2006.

47. S. Weis, S. Sarma, R. Rivest, and D. Engels. Security and privacy aspects of low-cost radio frequency identification systems. In Security in Pervasive Computing, pages 201-212, 2004

48. M. Weiser. The computer for the twenty-first century. Scientific American, 265(3):94-104, 1991.

49. M. Weiser. Some computer science issues in ubiquitous computing. Commun. ACM, 36(7):75-84, 1993.

50. M. Weiser and J. Brown. The coming age of calm technology. In P. Denning and R. Metcalfe, editors, Beyond calculation: The next fifty years of computing. New York, Copernicus, 1998.

51. M. Weiser, R. Gold, and J. Brown. The origins of ubiquitous computing research at PARC in the late 1980s. IBM Systems Journal, 38(4):693-696, 1999.

52. Y. Xiao, S. Yu, K. Wu, Q. Ni, C. Janecek, and J. Nordstad Radio frequency identification: technologies, applications, and research issues: Research articles. Wireless Communications \& Mobile Computing, 7(4):457-472, 2007.

Wirel. Commun. Mob. Comput. si: 1-15 (2008) 